\title{
Dual-mode immunoassay system based on glucose oxidase-triggered Fenton reaction for qualitative and quantitative detection of danofloxacin in milk
}

\author{
Suhua Wang, $\odot$ Bolong Fang, Meifang Yuan, Zexiang Wang, Juan Peng, and Weihua Lai* \\ State Key Laboratory of Food Science and Technology, Nanchang University, Nanchang 330047, China
}

\section{ABSTRACT}

In this study, a novel colorimetric and fluorescent dual-mode ELISA based on glucose oxidase (GOx)triggered Fenton reaction was developed for the qualitative and quantitative detection of danofloxacin (DAN). In this system, streptavidin-linked biotinylated anti-DAN-monoclonal antibody (SA-Bio-mAb) and biotinylated GOx (Bio-GOx) form the immune complex mAb-Bio-SA-Bio-GOx. In the absence of DAN, the mAb-Bio-SA-Bio-GOx would be immobilized by combining with coated DAN-BSA and catalyzed glucose to generate $\mathrm{H}_{2} \mathrm{O}_{2}$. The Fenton reaction between $\mathrm{H}_{2} \mathrm{O}_{2}$ and $\mathrm{Fe}^{2+}$ generated hydroxyl radicals, which oxidized the $o$-phenylenediamine to 2,3-diamino-phenazine. A dualsignal immunoassay with colorimetry and fluorescence as the signal readout was established. In the presence of DAN, DAN and DAN-BSA competed with Bio-mAb, decreasing the connection between immune complexes and DAN-BSA and finally resulting in lower signal of colorimetry and fluorescence. Under optimal conditions, the limit of detection of the fluorescence immunoassay was $0.337 \mathrm{ng} / \mathrm{mL}$ and was 5.24 -fold lower than that of traditional ELISA. The colorimetric immunoassay cutoff value was $30 \mathrm{ng} / \mathrm{mL}$ in milk. The average recoveries of the method for milk samples that are spiked with different concentrations of DAN were 91.1 to $128.3 \%$, with a coefficient of variation of 0.7 to $8.2 \%$. These results of the method exhibited good agreement with those of liquid chromatography-tandem mass spectrometry system (LC-MS/MS) method. In brief, this work provides an improved screening strategy with high sensitivity and accuracy for the qualitative or quantitative detection of DAN in milk monitoring.

Key words: colorimetry and fluorescence, ELISA, qualitative and quantitative detection, danofloxacin

Received January 24, 2020.

Accepted April 18, 2020.

*Corresponding author: talktolaiwh@163.com

\section{INTRODUCTION}

Danofloxacin (DAN) is a third-generation animalspecific fluoroquinolone with wide antibacterial spectrum and strong bactericidal activity (Aliabadi and Lees, 2003). It has high concentration in animal lung tissue and good antibacterial effect on respiratory pathogens (Tian et al., 2019). According to Announcement No. 235, Maximum Veterinary Drug Residue in Animal Food, of the Ministry of Agriculture of China (2002), the maximum residue limit of DAN in milk was set at $30 \mathrm{ng} / \mathrm{mL}$.

In milk, DAN has been detected by various analytical methods, including liquid chromatography (LC) with fluorescence detection (Zhang et al., 2017), LC-tandem mass spectrometry (LC-MS/MS; Moreno-González et al., 2014). However, these apparatus methods require expensive equipment, complicated pretreatment processes, and skillful operators (Wen et al., 2012).

A rapid, convenient, and low-cost method for qualitative and quantitative detection of DAN needs to be established. The most popular technique is ELISA, due to its high throughput and low cost (Rissin et al., 2010). However, the traditional ELISA has a low sensitivity (Sheng et al., 2009). Recently, glucose oxidase (GOx), which catalyzes glucose to produce $\mathrm{H}_{2} \mathrm{O}_{2}$, has been widely used in biosensors (Leskovac et al., 2005; Xiong et al., 2018). The mixture of $\mathrm{Fe}(\mathrm{II})-\mathrm{H}_{2} \mathrm{O}_{2}$ is regarded as the Fenton reagent. This mixture has remarkable oxidation capability, and it is widely used in environmental chemistry for degrading organic pollutants of wastewater (Boulangé et al., 2019). More research has shown that the oxidative effect of the Fenton reagent is heavily related to the hydroxyl radicals $\left({ }^{\bullet} \mathbf{O H}\right)$ referred as intermediated (Lacasa et al., 2019). Recently, colorimetric assay of ${ }^{\bullet} \mathrm{OH}$ based on the formation of electroactivity or optical active substrate induced by Fenton reaction has been reported (Jiao et al., 2017; Lai et al., 2017; Peng et al., 2019). The Fenton reaction progressed with accompanying occurrence of enzyme catalyst reaction, and then the obtained ${ }^{\bullet} \mathrm{OH}$ effectively initiated the catalytic oxidation of $o$-phenylenediamine 
(OPD) to 2,3-aminoazobenzene (DAP), resulting in the production of colorimetric and fluorescence signals.

In the present work, an ELISA method was proposed using GOx to oxidize glucose to produce $\mathrm{H}_{2} \mathrm{O}_{2}$, and then $\mathrm{Fe}^{2+}$ was added to generate ${ }^{\circ} \mathrm{OH}$, which can accelerate the self-oxidation from OPD to DAP with the change of colorimetry and fluorescence. Colorimetric and fluorescence signals are used for qualitative and quantitative detection of DAN, respectively. The proposed method may provide an improved screening strategy with high sensitivity and accuracy.

\section{MATERIALS AND METHODS}

\section{Materials and Equipment}

Bovine serum albumin, Tween 20, streptavidin (SA), D-(+)-glucose, biotin-3-sulfo-N-hydro-hydroxysuccinimide ester sodium salt, and GOx were from SigmaAldrich Chemical Co. (St. Louis, MO). We purchased OPD from Aladdin Biochemical Technology Co. Ltd. (Shanghai, China) and acquired DAN, ofloxacin (OFL), lomefloxacin (LOM), gatifloxacin (GAT), cinoxacin (CIN), fleroxacin (FLR), ciprofloxacin (CIP), enrofloxacin (ENR), marbofloxacin (MAR), sarafloxacin (SAR), sulfamethazine (SMZ), and olaquindox (OLA) from Yuan Ye Biotechnology Co. Ltd. (Shanghai, China). The 96-well ELISA microplates were bought from ExCell Bio (Shanghai, China). Black 96-well microplates were purchased from Corning Inc. (Corning, NY). We purchased DAN-monoclonal antibody (mAb) from Wuxi Zodoboer Biotech Co. Ltd. (Wuxi, China). Horseradish peroxidase (HRP)-labeled goat anti-mouse immunoglobulin was purchased from Shanghai Universal Biotech Co. Ltd. (Shanghai, China). Negative milk samples, confirmed by LC-MS/MS, and 20 real milk samples were provided by Jiangxi Provincial Veterinary Medicine Feed Supervision Institute (Nanchang, Jiangxi, China). All other chemicals and reagents were of analytical grade and were purchased from Sinopharm Chemical Reagent Co. Ltd. (Shanghai, China). The LC-MS/MS system was composed of a triple-quadrupole instrument (Agilent 6410) and an LC system (Agilent 1200 series; Agilent Technologies, Lexington, MA). A Varioskan LUX multimode microplate reader was purchased from Thermo Fisher Scientific Co. (Waltham, MA) to measure the fluorescence signal. The commercialized ELISA kit was purchased from Reagen Co. Ltd. (Moorestown, NJ).

\section{Preparation of Bio-mAb and Bio-GOx}

The Bio-mAb and Bio-GOx were synthesized as described previously with some modifications (Xiong et al., 2018). For Bio-mAb preparation, $1 \mathrm{~mL}$ of $\mathrm{mAb}$ $(1 \mathrm{mg} / \mathrm{mL})$ was added into $750 \mu \mathrm{L}$ of biotin-3-sulfo- $\mathrm{N}$ hydroxysuccinimide ester sodium salt $(1 \mathrm{mg} / \mathrm{mL})$ and $250 \mu \mathrm{L}$ of PBS (0.01 M, pH 7.4) and then stirred at room temperature for $3 \mathrm{~h}$. The reacting mixture was dialyzed in PBS (0.01 $M, \mathrm{pH} 7.4)$ for $72 \mathrm{~h}$. For Bio-GOx preparation, $6.5 \mathrm{~mL}$ of GOx $(4.38 \mathrm{mg} / \mathrm{mL})$ was added to $600 \mu \mathrm{L}$ of biotin-3-sulfo-N-hydroxysuccinimide ester sodium salt $(1.7 \mathrm{mg} / \mathrm{mL})$ and stirred at room temperature for $2 \mathrm{~h}$. The reacting mixture was dialyzed in PBS (0.01 M, pH 7.4) for $72 \mathrm{~h}$.

\section{Proposed ELISA Procedure}

Coated antigen and Bio-mAb concentrations were first optimized using a chessboard design. The coated antigen was diluted into $2,1.5,1,0.75,0.5,0.25$, and $0.1 \mu \mathrm{g} / \mathrm{mL}$ in carbonate buffer solution $(0.01 M, \mathrm{pH}$ 9.6). Then, $100 \mu \mathrm{L}$ of the diluted solution was added to ELISA wells and incubated at $37^{\circ} \mathrm{C}$ for $2 \mathrm{~h}$. The ELISA wells were washed with a PBS and Tween solution (PBST; $0.01 M$ PBS containing 0.05\% Tween 20, vol/vol) 3 times. The ELISA wells were blocked with $260 \mu \mathrm{L}$ of blocking buffer $(0.01 M$ carbonate buffer solution containing $0.2 \%$ gelatin) and then incubated at $4^{\circ} \mathrm{C}$ overnight. After the ELISA wells were washed with PBST 3 times, $50 \mu \mathrm{L}$ of standard solution and 50 $\mu \mathrm{L}$ of Bio-mAb $(2,1.5,1,0.75,0.5,0.25$, and $0.1 \mu \mathrm{g} /$ $\mathrm{mL}$ ) were sequentially added to each well. After being incubated at $37^{\circ} \mathrm{C}$ for 30 min, the ELISA wells were washed with PBST 3 times, and then $100 \mu \mathrm{L}$ of SA was added to each well, and they were incubated at $37^{\circ} \mathrm{C}$ for $30 \mathrm{~min}$. After the plates were washed with PBST 3 times, $100 \mu \mathrm{L}$ of Bio-GOx was added, and they were then incubated at $37^{\circ} \mathrm{C}$ for $30 \mathrm{~min}$. After the plates were washed with PBST 3 times, $100 \mu \mathrm{L}$ of glucose dissolved in PBS was added to each well, and they were incubated at $37^{\circ} \mathrm{C}$ for $1 \mathrm{~h}$. Thereafter, $50 \mu \mathrm{L}$ of $\mathrm{Fe}^{2+}$ and $50 \mu \mathrm{L}$ of OPD were added to the ELISA wells. The ELISA wells were then read at $487 \mathrm{~nm}$ for the optical density value $\left(\mathrm{OD}_{487}\right)$ and at $569 \mathrm{~nm}$ for the fluorescence intensity (excited wavelength was $417 \mathrm{~nm}$ ).

\section{Optimization of Proposed ELISA}

To obtain optimal parameters, the study analyzed the different concentrations of the following: SA $(0.05$, $0.1,0.25,0.51,2.5$, and $5 \mu \mathrm{g} / \mathrm{mL})$, Bio-GOx $(0.05$, $0.1,0.5,1.0$, and $2.0 \mu \mathrm{g} / \mathrm{mL})$, glucose $(0,1,5,10,25$, and $50 \mathrm{mM}), \mathrm{Fe}^{2+}(0.01,0.05,0.1,0.5,1,5,10$, and 25 $\mathrm{m} M)$, and $\mathrm{OPD}(0,1,2.5,5,10,25$, and $50 \mathrm{mM})$, as well as the $\mathrm{pH}$ value of the Fenton system $(0.5,1,2,3$, 4 , and 5). 


\section{Detection of DAN in PBS}

The DAN standard solutions were prepared by diluting the DAN stock solution with PBS (0.01 $M, \mathrm{pH} 7.4)$ to different DAN concentrations $(0,0.1,0.25,0.5,1$, $2.5,5,10,25,50$, and $100 \mathrm{ng} / \mathrm{mL}$ ). Each DAN standard solution was determined three times by the proposed ELISA. The response curve was established by plotting fluorescence signal intensity against the concentration of DAN.

\section{Specificity of Proposed ELISA}

The specificity of our proposed method was evaluated by analyzing DAN, DAN structural analogs (OFL, LOM, GAT, ENR, MAR, SAR, CIN, FLR, and CIP), SMZ, and OLA. All drug concentrations were $10 \mathrm{ng} /$ $\mathrm{mL}$, and all experiments were repeated 3 times.

\section{LC-MS/MS Analysis}

The proposed ELISA for detection of DAN was further confirmed by a triple-quadrupole LC-MS/MS system (Agilent Technologies). The sample was pretreated according to Chinese National Standard, 2008 (GB/T22985-2008). Milk samples (2.0 g) and $10 \mathrm{~mL}$ of acetonitrile were added to a $50-\mathrm{mL}$ centrifuge tube and shaken with a vortex mixer for $1 \mathrm{~min}$. After centrifugation at $11,040 \times g$ for $5 \mathrm{~min}$ at room temperature, the supernatant was transferred to another centrifuge tube and evaporated at $50^{\circ} \mathrm{C}$. Five milliliters of PBS $(0.05$ $M, \mathrm{pH} 3$ ) was added to the centrifuge tube, shaken with a vortex mixer for $1 \mathrm{~min}$, purified by solid-phase extraction column, and then filtered with a $0.22-\mu \mathrm{m}$ cellulose membrane. The LC-MS/MS system was controlled by Mass Hunter software (Agilent Technologies). The chromatographic separation was performed with an Agilent Zorbax Eclipse XDB $\mathrm{C}_{18}$ column $(150 \mathrm{~mm} \times 2.1 \mathrm{~mm}$, $5 \mu \mathrm{m})$ maintained at $30^{\circ} \mathrm{C}$. The mobile phase consisted of solvent A ( $0.1 \%$ acetic acid water) and solvent B (methanol). The initial solvent gradient condition was set at $80 \%$. The linear gradient decreased by $40 \%$ from 0 to $5.0 \mathrm{~min}$, and then remained unchanged from 5.0 min to $9.0 \mathrm{~min}$. Starting at $9.10 \mathrm{~min}$, the gradient was set to rebalance the column under initial conditions to $11.0 \mathrm{~min}$. The flow rate was $0.20 \mathrm{~mL} / \mathrm{min}$, and the injection volume was $15 \mu \mathrm{L}$ in full circulation. Ionization was achieved using electrospray ionization in positive ion mode. Detection was conducted in multiple reaction monitoring mode and dissolved with high-purity nitrogen, cone gas, and collision gas. The monitoring ion pairs were chosen as DAN $m / z 358.14 / 340.00$ (quantitative ion) and 358.14/339.83 (qualitative ions).

\section{Detection of Milk}

The negative samples of milk confirmed by LC-MS/ MS were spiked with DAN at 10, 20, 30, and $40 \mathrm{ng} / \mathrm{mL}$ for qualitative analysis and at $2.5,5,10$, and $25 \mathrm{ng} / \mathrm{mL}$ for quantitative analysis. The accuracy and precision of the proposed ELISA were estimated by calculating their recovery ratio and coefficient of variation $(\mathbf{C V})$ in quantitative analysis. Each measurement was performed thrice. Twenty real milk samples were determined using the proposed ELISA and HPLC-MS/MS.

\section{RESULTS AND DISCUSSION}

\section{Principle of the Proposed ELISA}

Figure 1 illustrates the proposed ELISA. In the absence of DAN, Bio-mAb was captured by DAN-BSA that is immobilized on the microplates. After addition of SA and Bio-GOx, the mAb-Bio-SA-Bio-GOx complex was formed. Produced by the oxidation of glucose under the catalysis of $\mathrm{GOx}, \mathrm{H}_{2} \mathrm{O}_{2}$ reacts with $\mathrm{Fe}^{2+}$ to produce ${ }^{\bullet} \mathrm{OH}$. The ${ }^{\bullet} \mathrm{OH}$ then oxidizes $\mathrm{OPD}$ to DAP, with a yellow color and fluorescence. In the presence of DAN, GOx was not immobilized on the microplates and could not produce $\mathrm{H}_{2} \mathrm{O}_{2}$. The OPD could not be oxidized to DAP with colorimetric and fluorescent signals.

\section{Analysis of Optical Characterization in OPD and DAP}

Figure 2 shows an optical characterization of OPD and DAP. The UV-visible absorption peak of DAP was $487 \mathrm{~nm}$. However, OPD did not have a UV-visible absorption peak (Figure 2A). The maximum fluorescent excitation and maximum fluorescent emission wavelength of DAP were $417 \mathrm{~nm}$ and $569 \mathrm{~nm}$, respectively. However, OPD did not have fluorescent excitation and emission peaks (Figure 2B). A large stoke shift $(>150$ $\mathrm{nm}$ ) is evident for DAP and can effectively eliminate the interference of various nonspecific fluorescence, leading to high sensitivity. The absorption intensity and fluorescence intensity of DAP are significantly enhanced compared with those of OPD. Moreover, the oxide (DAP) of OPD had a significant colorimetric change. Colorimetry and fluorescence of DAP could be used as signal outputs for the proposed method.

\section{Optimization of Key Parameters}

The concentrations of coated antigen and Bio-mAb were optimized using a chessboard method. When the concentration of coated antigen and Bio-mAb were 


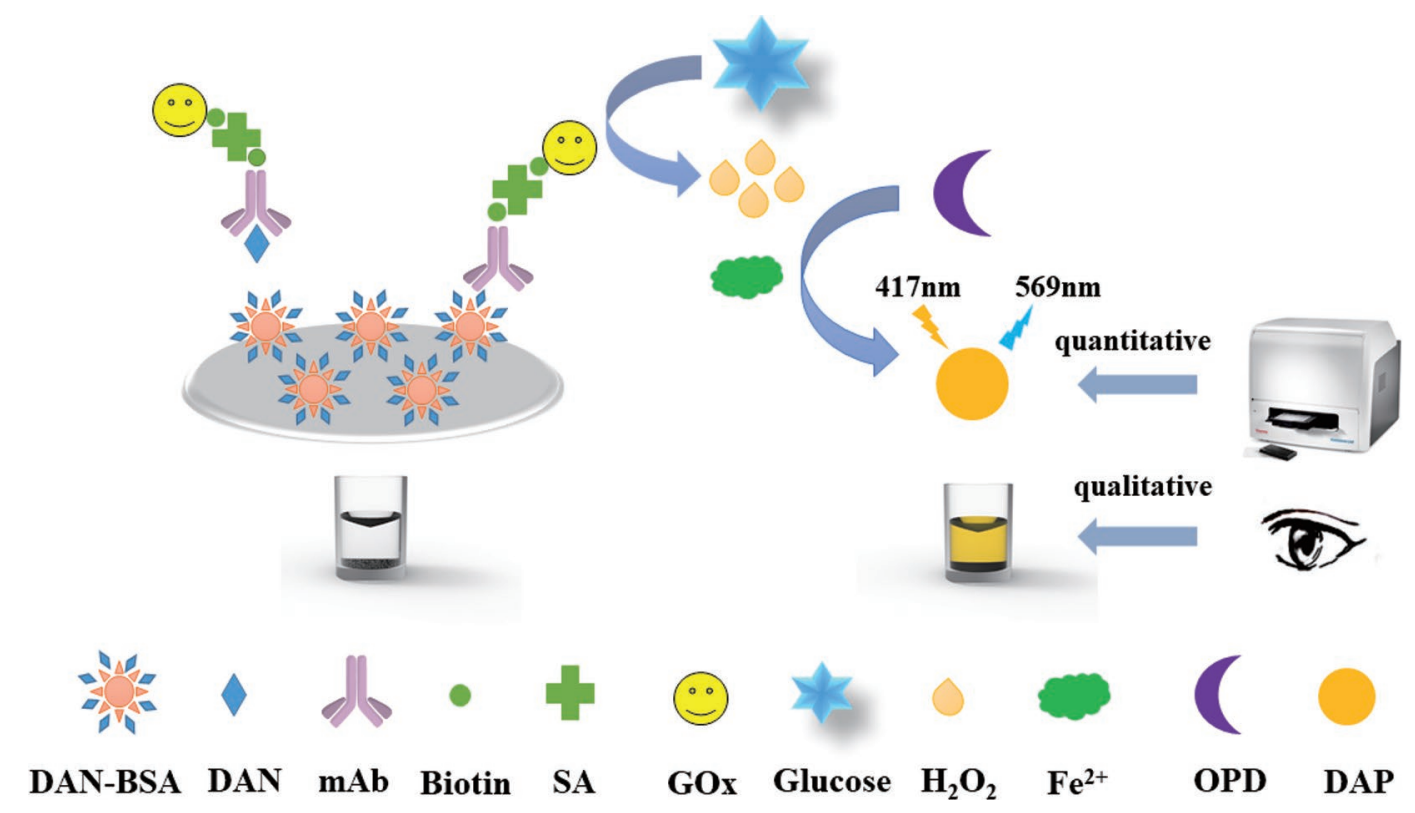

Figure 1. Schematic of detection of danofloxacin (DAN) by a dual-mode signal output system based on glucose oxidase (GOx)-triggered Fenton reaction ELISA. $\mathrm{mAb}=$ monoclonal antibody; $\mathrm{SA}=$ streptavidin; $\mathrm{OPD}=o$-phenylenediamine; $\mathrm{DAP}=2,3$-aminoazobenzene.

1 and $0.75 \mu \mathrm{g} / \mathrm{mL}$, the fluorescence intensity of the DAN-negative sample was higher than those of others (Supplemental Table S1, https://doi.org/10.3168/jds .2020-18256). Therefore, the optimum concentrations of coated antigen and Bio-mAb were selected at 1 and $0.75 \mu \mathrm{g} / \mathrm{mL}$, respectively. Figure 3A shows the effect of SA concentration on fluorescence intensity. When the concentration of SA was increased from $0.05 \mu \mathrm{g} /$ $\mathrm{mL}$ to $1.0 \mu \mathrm{g} / \mathrm{mL}$, the fluorescence intensity gradually increased from 0.32 arbitrary units (a.u.) to 5.38 a.u.. As the concentration of SA was increased to $5 \mu \mathrm{g} /$ $\mathrm{mL}$, the fluorescence intensity remained steady at approximately 5.38 a.u. Thus, the optimal concentration of SA was $1.0 \mu \mathrm{g} / \mathrm{mL}$. Figure 3B shows the effect of the concentration of Bio-GOx on fluorescent intensity. When the concentration of Bio-GOx was increased from $0.05 \mu \mathrm{g} / \mathrm{mL}$ to $0.5 \mu \mathrm{g} / \mathrm{mL}$, the fluorescence intensity gradually rose to 5.53 a.u. When the concentration of Bio-GOx was increased to $2 \mu \mathrm{g} / \mathrm{mL}$, the fluorescence intensity continued at approximately 5.53 a.u. To obtain a higher fluorescence intensity, we selected $0.5 \mu \mathrm{g} /$ $\mathrm{mL}$ as the optimal concentration of Bio-GOx. Figure $3 \mathrm{C}$ shows the effect of the concentration of glucose on fluorescence intensity. The fluorescence intensity increased from 0.07 to 5.20 a.u. when glucose concentration was increased from 1 to $25 \mathrm{mM}$. When the
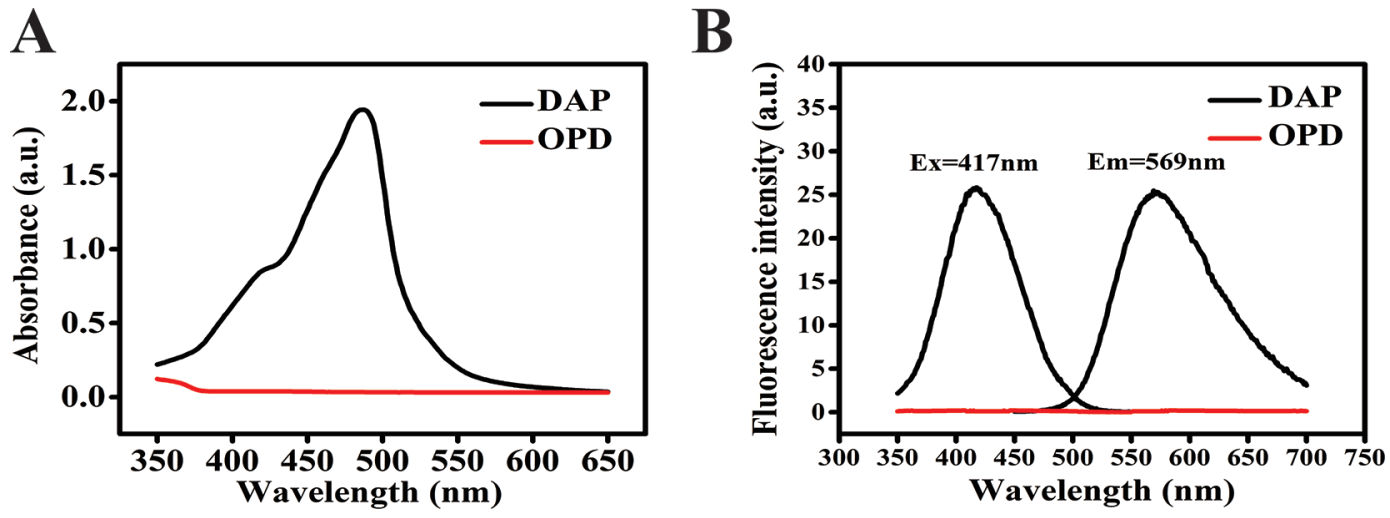

Figure 2. Optical characterization of 2,3-aminoazobenzene (DAP) and o-phenylenediamine (OPD). (A) UV-visible absorption spectrum of DAP and OPD. (B) Excitation (Ex) and emission (Em) spectra of DAP and OPD. a.u. = arbitrary units. 
concentration of glucose was increased to $50 \mathrm{~m} M$, the fluorescence intensity remained at approximately 5.20 a.u. Thus, the optimal concentration of glucose was 25 $\mathrm{m} M$. Figure 3D shows the effect of the concentration of $\mathrm{Fe}^{2+}$ on fluorescence intensity. The concentration of $\mathrm{Fe}^{2+}$ was increased from 0.01 to $10.0 \mathrm{mM}$, and the fluorescence intensity gradually increased to 5.00 a.u. As the concentration of $\mathrm{Fe}^{2+}$ was increased to $25 \mathrm{mM}$, the fluorescence intensity remained stable. Therefore, the optimal concentration of $\mathrm{Fe}^{2+}$ was $10 \mathrm{~m} M$. Figure $3 \mathrm{E}$ shows the effect of $\mathrm{pH}$ on fluorescence intensity. When the $\mathrm{pH}$ value was in the range of 0.5 to 1.0 , the fluorescence intensity remained stable at approximately 5.68 a.u. Subsequently, as the $\mathrm{pH}$ was increased to 5 , the fluorescence intensity decreased to 0.9 a.u. Therefore, the optimal $\mathrm{pH}$ was 1 . These results implied that a strong acid $\mathrm{pH}$ was beneficial for achieving a higher fluorescence intensity for DAP. However, the $\mathrm{pH}$ was lower than that reported in most studies (Mijangos et al., 2006; Abbas et al., 2010). The reason might be that the OPD was easy to oxidize, and we controlled the response time within $5 \mathrm{~min}$. The strong acid $(\mathrm{pH}$ 1) was beneficial for achieving a higher fluorescence intensity for DAP in a short time. Thus, the optimal $\mathrm{pH}$ was selected at 1 . Figure $3 \mathrm{~F}$ shows the effect of OPD concentration on fluorescence intensity. When the concentration of OPD was increased from 1 to $10 \mathrm{mM}$, the fluorescence intensity gradually increased to 5 a.u. With further increase of OPD concentration from 10 to
$50 \mathrm{~m} M$, fluorescence intensity remained stable. Therefore, the optimal concentration of OPD was $10 \mathrm{~m} M$.

\section{Standard Curve for DAN of Proposed ELISA}

The standard curves of the proposed ELISA based on colorimetric and fluorescent intensity in PBS were established. Figure 4 shows the calibration curve constructed by plotting the fluorescence intensity against the logarithm of DAN concentration (0 to $100 \mathrm{ng} / \mathrm{mL}$ ). The linear equation of the proposed method for quantitative detection of DAN can be described as $\mathrm{Y}=-2.756$ $\lg \mathrm{X}+4.480\left(R^{2}=0.997\right)$, with a good linear detection range from $1 \mathrm{ng} / \mathrm{mL}$ to $25 \mathrm{ng} / \mathrm{mL}$. In the equation, $\mathrm{Y}$ is the fluorescence intensity and $\mathrm{X}$ is the DAN concentration. The limit of detection (LOD), defined as the blank signal minus 3 standard deviations (Guo et al., 2016), was calculated as $0.337 \mathrm{ng} / \mathrm{mL}$, indicating that LOD of the fluorescence immunoassay was 5.24-fold lower than that of conventional ELISA (Supplemental Figure S1, https://doi.org/10.3168/jds.2020-18256). Supplemental Figure S2 (https://doi.org/10.3168/jds .2020-18256) shows that the linear equation based on colorimetric signal for quantitative detection of DAN was $\mathrm{Y}=-0.603 \lg \mathrm{X}+0.786\left(\mathrm{R}^{2}=0.993\right)$, with a good linear detection range from 1 to $10 \mathrm{ng} / \mathrm{mL}$. The LOD was $0.684 \mathrm{ng} / \mathrm{mL}$. Supplemental Table S2 (https: //doi.org/10.3168/jds.2020-18256) exhibits the LOD of this method and others. This result indicated that the
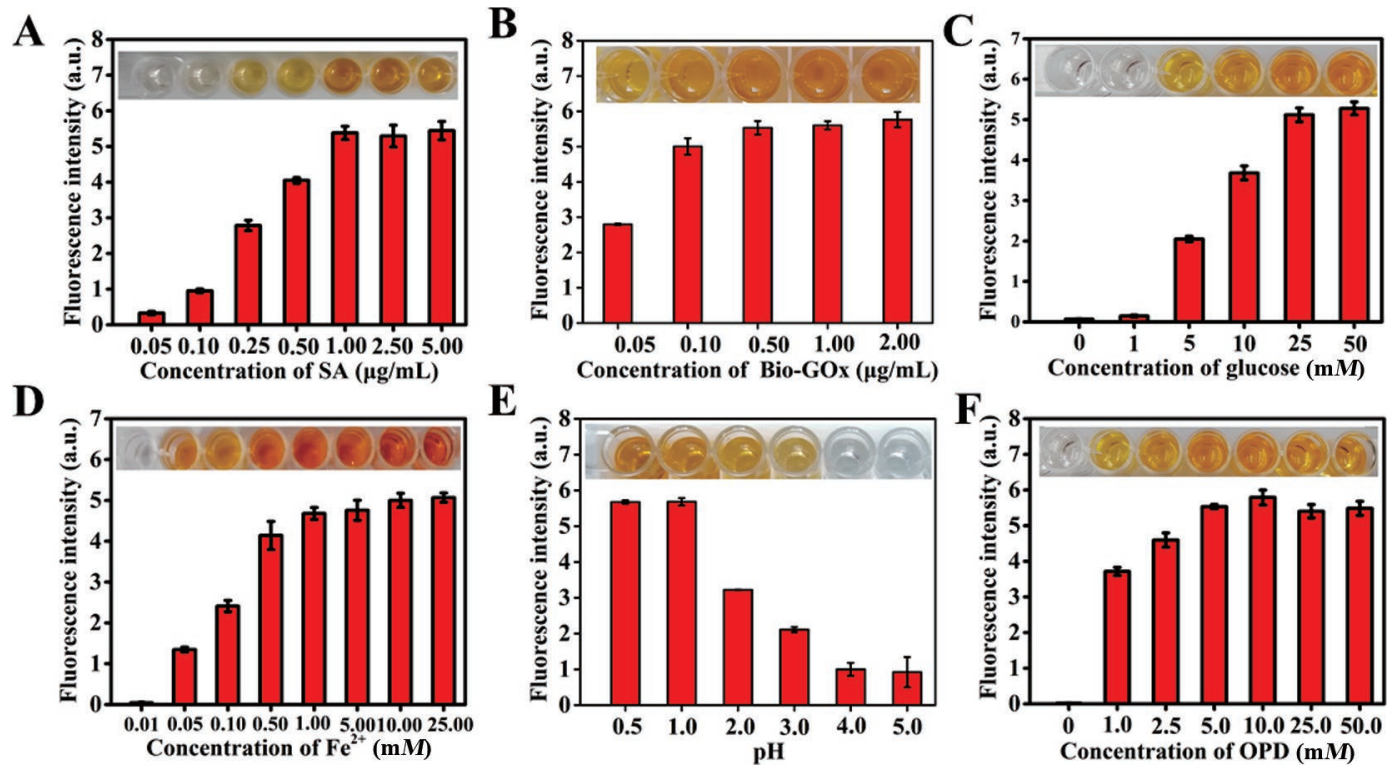

Figure 3. Parameter optimization in proposed ELISA. (A) Streptavidin concentration (SA, 0.05-5 $\mu \mathrm{g} / \mathrm{mL}$ ); (B) biotinylated glucose oxidase concentration (Bio-GOx, 0.05-2 $\mu \mathrm{g} / \mathrm{mL}) ;(\mathrm{C})$ glucose concentration $(0-50 \mathrm{mM})$; (D) Fe ${ }^{2+}$ concentration $(0.01-25 \mathrm{mM})$; (E) pH $(0.5-5)$; and (F) o-phenylenediamine concentration (OPD, 0-50 $\mathrm{m} M)$. Error bars indicate $\mathrm{SD}(\mathrm{n}=3)$. 


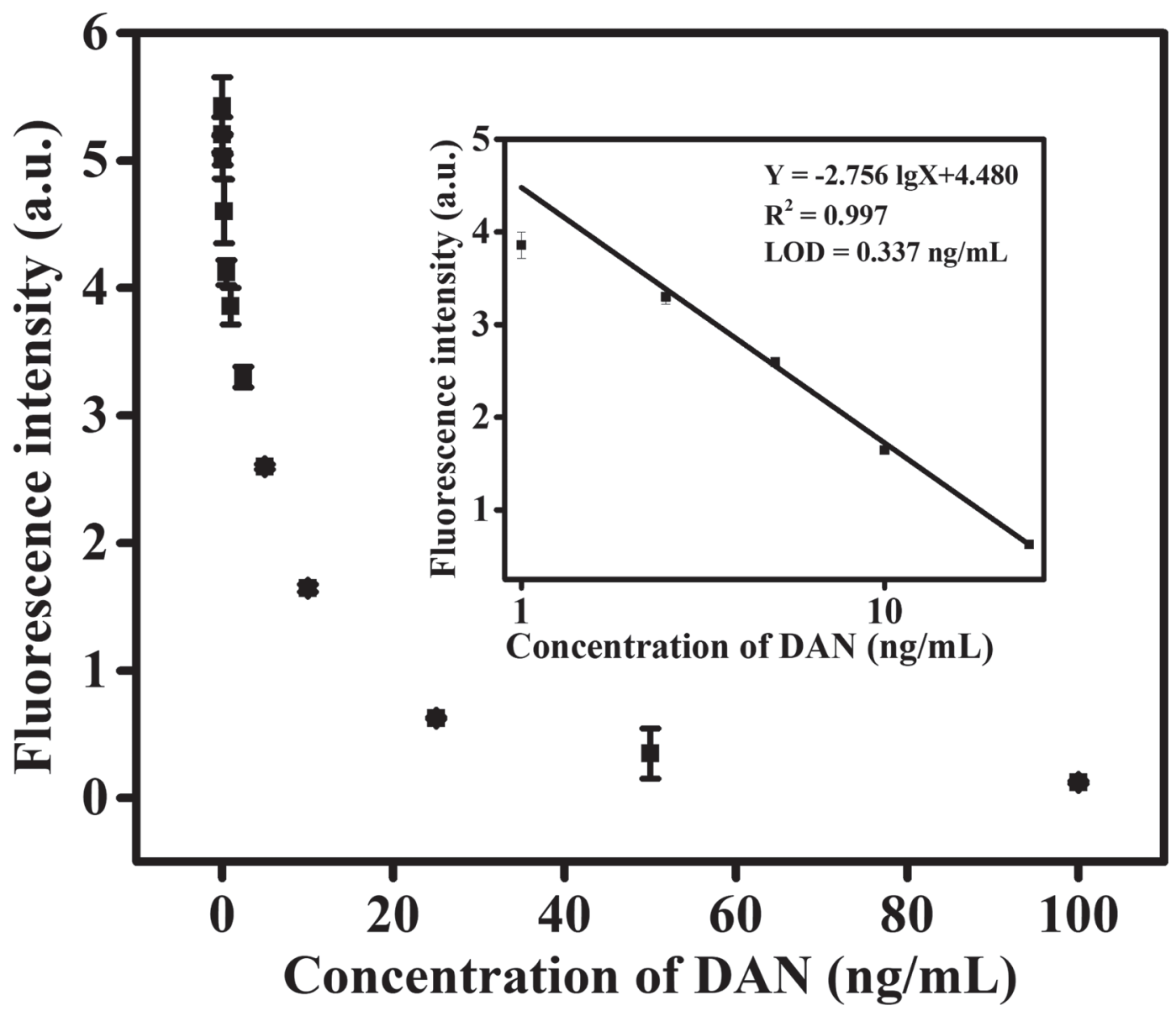

Figure 4. Calibration curve of proposed fluorescence ELISA. The danofloxacin (DAN) concentration ranges from 0 to $100 \mathrm{ng} / \mathrm{mL}$. The inset shows a linear range of 1 to $25 \mathrm{ng} / \mathrm{mL}$. LOD $=$ limit of detection. Error bars $=\mathrm{SD}$.

LOD of the proposed method was lower than that of the other methods listed in Supplemental Table S2 and could be used for quantitative detection of DAN. The LOD of our proposed ELISA was more sensitive than that of the commercialized ELISA kit (Supplemental Figure S3, https://doi.org/10.3168/jds.2020-18256). When OPD was added as enzyme substrate in the commercialized ELISA kit, the LOD was higher than that of the proposed ELISA (Supplemental Figure S4, https: //doi.org/10.3168/jds.2020-18256).

\section{Specificity of Proposed ELISA}

The specificity of this method was evaluated by analyzing DAN, DAN structural analogs (OFL, LOM, GAT, ENR, MAR, SAR, CIN, FLR, and CIP), SMZ, and OLA. All drug concentrations were $10 \mathrm{ng} / \mathrm{mL}$. Figure 5 shows that the fluorescence intensity of other drugs was approximately 5 a.u., whereas the fluorescence intensity of DAN was 1.58 a.u. This result indicats that the proposed ELISA has good specificity.

\section{Accuracy and Precision of Proposed ELISA}

Figure 6 shows the negative samples of milk confirmed by LC-MS/MS spiked with DAN at 10, 20, 30 , and $40 \mathrm{ng} / \mathrm{mL}$ for qualitative analysis. The cutoff value was $30 \mathrm{ng} / \mathrm{mL}$ by naked-eye detection. The accuracy and precision of the proposed ELISA were further compared with those of the LC-MS/MS method by detecting $4 \mathrm{DAN}$-spiked milk samples. The recovery of fluorescence ELISA was 95.3 to $125 \%$ with CV of 1.9 to $8.2 \%$. The recovery of HPLC-MS/MS was 73.1 to $118 \%$, with CV of 0.7 to $6.3 \%$ (Table 1). These results indicate an acceptable accuracy and precision of the proposed ELISA for the quantitative detection of DAN in actual milk samples. Supplemental Table S3 (https: //doi.org/10.3168/jds.2020-18256) shows that 1 real milk sample was positive (sample 6) and 19 real milk samples were negative for presence of DAN using the proposed ELISA and LC-MS/MS. The detection result of the ELISA was compatible with that of the HPLCMS/MS method. 


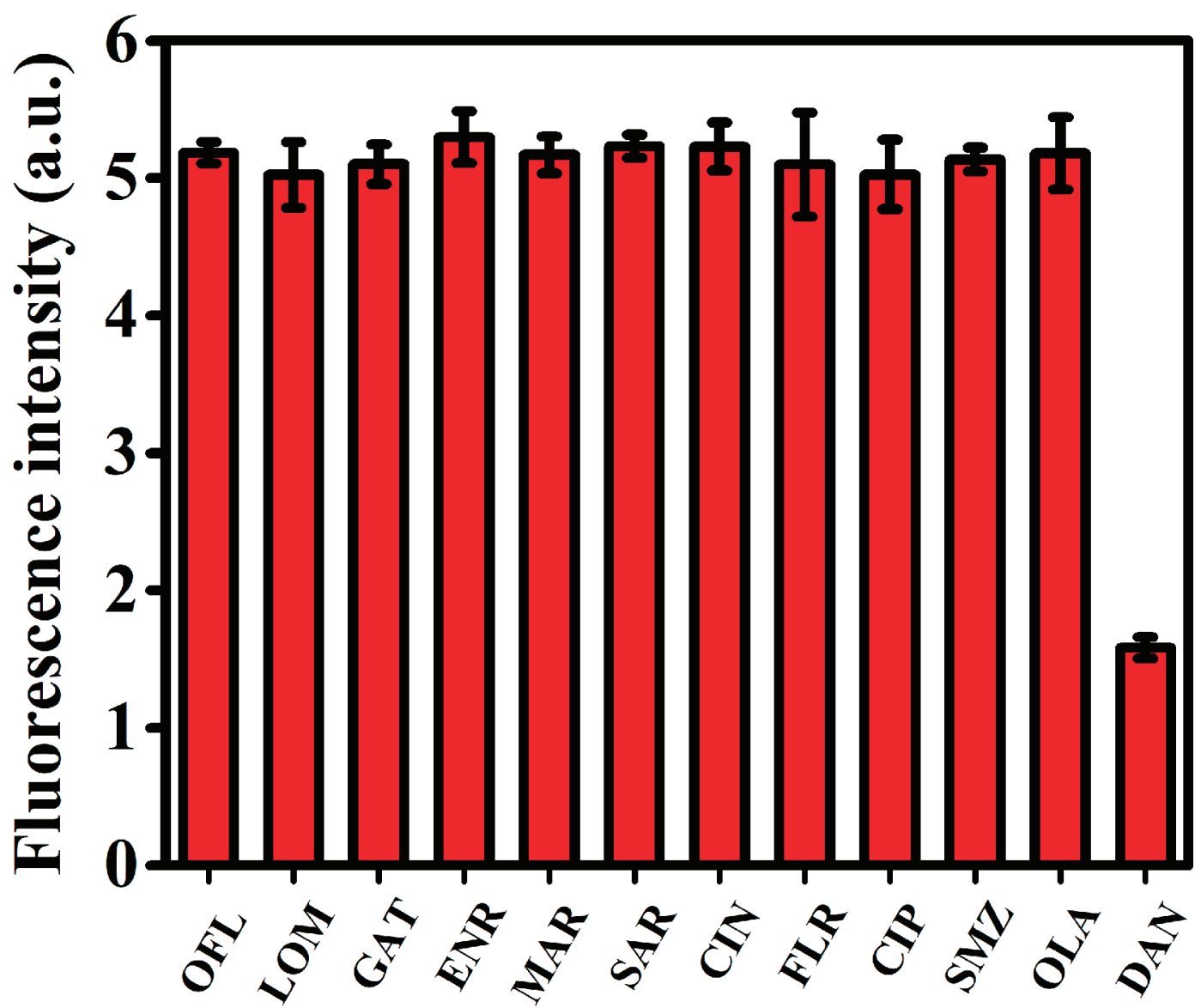

Figure 5. Specificity of this method in detecting danofloxacin (DAN) with 9 other quinolones [ofloxacin (OFL), lomefloxacin (LOM), gatifloxacin (GAT), enrofloxacin (ENR), marbofloxacin (MAR), sarafloxacin (SAR), cinoxacin (CIN), fleroxacin (FLR), and ciprofloxacin (CIP)], sulfamethazine (SMZ), and olaquindox (OLA). a.u. = arbitrary units. Error bars indicate SD $(\mathrm{n}=3)$.

\section{CONCLUSIONS}

In this study, a novel colorimetric and fluorescent dual-mode immunoassay system was developed based on GOx-triggered Fenton reaction for qualitative and quantitative detection of DAN. The cut-off value was $30 \mathrm{ng} / \mathrm{mL}$ by naked-eye detection. The proposed fluorescent ELISA demonstrates a high sensitivity for DAN detection, with LOD of $0.337 \mathrm{ng} / \mathrm{mL}$, which is ap-

\section{$\begin{array}{llll}\text { A } & \text { B } & \text { C } & \text { D }\end{array}$}

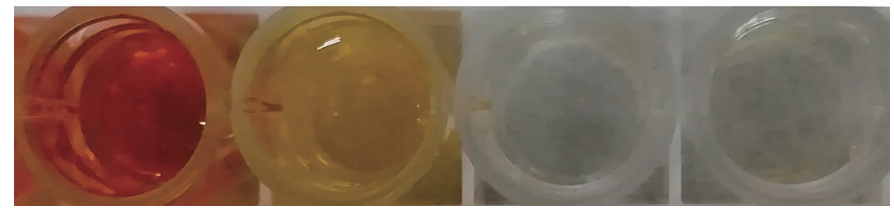

Figure 6. Colorimetric immunoassay in milk at different danofloxacin (DAN)-spiked concentrations. (A) $10 \mathrm{ng} / \mathrm{mL}$, (B) $20 \mathrm{ng} / \mathrm{mL}$, (C) $30 \mathrm{ng} / \mathrm{mL}$, and (D) $40 \mathrm{ng} / \mathrm{mL}$. proximately 5.24-fold lower than that of a conventional ELISA. The average recoveries for milk samples spiked with different concentrations of DAN ranged from 91.1 to $128 \%$, with a CV that ranged from 0.7 to $8.2 \%$. The accuracy and precision of the proposed method was further confirmed using the LC-MS/MS method. The proposed ELISA method provides an improved screening strategy with high sensitivity for the qualitative or quantitative detection of DAN in milk monitoring.

\section{ACKNOWLEDGMENTS}

This work was supported by the National Key Research and Development Program of China (2019YFC1605502; Beijing), Program of State Key Laboratory of Food Science and Technology, Nanchang University (SKLF-ZZA-201912; Nanchang, China), and the free explore issue of State Key Laboratory of Food Science and Technology of Nanchang University (SKLF-ZZB-201913; Nanchang, China). The authors have not stated any conflicts of interest. 
Table 1. Comparison of addition and recovery of fluorescence ELISA and liquid chromatography-tandem mass spectrometry (LC-MS/MS) detection ${ }^{1}$

\begin{tabular}{lccccccc}
\hline & \multicolumn{3}{c}{$\begin{array}{c}\text { Fluorescence ELISA } \\
(\mathrm{n}=3)\end{array}$} & & \multicolumn{3}{c}{$\begin{array}{c}\text { LC-MS/MS } \\
(\mathrm{n}=3)\end{array}$} \\
\cline { 2 - 3 } \cline { 6 - 8 } $\begin{array}{l}\text { DAN added } \\
(\mathrm{ng} / \mathrm{mL})\end{array}$ & $\begin{array}{c}\text { DAN } \\
(\mathrm{ng} / \mathrm{mL})\end{array}$ & $\begin{array}{c}\text { Recovery } \\
(\%)\end{array}$ & $\begin{array}{c}\mathrm{CV} \\
(\%)\end{array}$ & & $\begin{array}{c}\text { DAN } \\
(\mathrm{ng} / \mathrm{mL})\end{array}$ & $\begin{array}{c}\text { Recovery } \\
(\%)\end{array}$ & $\begin{array}{c}\mathrm{CV} \\
(\%)\end{array}$ \\
\hline 2.5 & 2.445 & 97.8 & 5.7 & & 2.114 & 84.6 & 6.3 \\
5 & 6.233 & 125 & 8.2 & & 5.924 & 118 & 4.6 \\
10 & 9.529 & 95.3 & 5.4 & & 9.210 & 92.1 & 0.7 \\
25 & 24.869 & 99.5 & 1.9 & & 18.275 & 73.1 & 1.3 \\
\hline
\end{tabular}

${ }^{1} \mathrm{DAN}=$ danofloxacin.

\section{REFERENCES}

Abbas, M. E., W. Luo, L. Zhu, J. Zou, and H. Tang. 2010. Fluorometric determination of hydrogen peroxide in milk by using a Fenton reaction system. Food Chem. 120:327-331. https://doi.org/10 .1016/j.foodchem.2009.10.024.

Aliabadi, F. S., and P. Lees. 2003. Pharmacokinetic-pharmacodynamic integration of danofloxacin in the calf. Res. Vet. Sci. 74:247-259. https://doi.org/10.1016/S0034-5288(03)00005-5.

Boulangé, M., C. Lorgeoux, C. Biache, A. Saada, and P. Faure. 2019. Fenton-like and potassium permanganate oxidations of PAH-contaminated soils: Impact of oxidant doses on PAH and polar PAC (polycyclic aromatic compound) behavior. Chemosphere 224:437444. https://doi.org/10.1016/j.chemosphere.2019.02.108.

Chinese National Standard. 2008. GB/T22985-2008. Determination of enrofloxacin, danofloxacin, ciprofloxacin, sarafloxacin, orbifloxacin, difloxacin and marbofloxacin in milk and milk powder-LCMS-MS method. http://openstd.samr.gov.cn/bzgk/gb/newGbInfo $?$ hcno $=$ AF874C316028F815C62D40FCA43D001B

Guo, Q., J. J. Han, S. Shan, D. F. Liu, S. S. Wu, Y. H. Xiong, and W. H. Lai. 2016. DNA-based hybridization chain reaction and biotin-streptavidin signal amplification for sensitive detection of Escherichia coli $\mathrm{O}_{157}: \mathrm{H}_{7}$ through ELISA. Biosens. Bioelectron. 86:990-995. https://doi.org/10.1016/j.bios.2016.07.049.

Jiao, L., Z. J. Xu, W. W. Du, H. Li, and M. Yin. 2017. Fast preparation of polydopamine nanoparticles catalyzed by $\mathrm{Fe}^{2+} / \mathrm{H}_{2} \mathrm{O}_{2}$ for visible sensitive smartphone-enabled cytosensing. ACS Appl. Mater. Interfaces 9:28339-28345. https://doi.org/10.1021/acsami $.7 \mathrm{~b} 10564$.

Lacasa, E., P. Cañizares, F. C. Walsh, M. A. Rodrigo, and C. Poncede-León. 2019. Removal of methylene blue from aqueous solutions using an $\mathrm{Fe}^{2+}$ catalyst and in situ $\mathrm{H}_{2} \mathrm{O}_{2}$ generated at gas diffusion cathodes. Electrochim. Acta 308:45-53. https://doi.org/10.1016/j .electacta.2019.03.218.

Lai, W., Q. Wei, M. Xu, J. Zhuang, and D. Tang. 2017. Enzymecontrolled dissolution of $\mathrm{MnO}_{2}$ nanoflakes with enzyme cascade amplification for colorimetric immunoassay. Biosens. Bioelectron. 89:645-651. https://doi.org/10.1016/j.bios.2015.12.035.

Leskovac, V., S. Trivic, G. Wohlfahrt, J. Kandrac, and D. Pericin. 2005. Glucose oxidase from Aspergillus niger: The mechanism of action with molecular oxygen, quinones, and one-electron acceptors. Int. J. Biochem. Cell Biol. 37:731-750. https://doi.org/10 $.1016 /$ j.biocel.2004.10.014.

Mijangos, F., F. Varona, and N. Villota. 2006. Changes in solution color during phenol oxidation by Fenton reagent. Environ. Sci. Technol. 40:5538-5543. https://doi.org/10.1021/es060866q.

Ministry of Agriculture of the People's Republic of China. 2002. Announcement No. 235. The highest veterinary drug in animal food Residue limit [S]. http://www.moa.gov.cn/govpublic/SYJ/ 201006/t20100606_1535491.htm?keywords=.
Moreno-González, D., F. J. Lara, L. Gámiz-Gracia, and A. M. GarcíaCampaña. 2014. Molecularly imprinted polymer as in-line concentrator in capillary electrophoresis coupled with mass spectrometry for the determination of quinolones in bovine milk samples. J. Chromatogr. A 1360:1-8. https://doi.org/10.1016/j.chroma.2014 .07 .049 .

Peng, T., J. Y. Wang, S. L. Xie, K. Yao, P. M. Zheng, Y. B. Ke, and H. Y. Jiang. 2019. Label-free gold nanoclusters as quenchable fluorescent probes for sensing olaquindox assisted by glucose oxidasetriggered Fenton reaction. Food Addit. Contam. Part A Chem. Anal. Control Expo. Risk Assess. 36:752-761.

Rissin, D. M., C. W. Kan, T. G. Campbell, S. C. Howes, D. R. Fournier, L. Song, T. Piech, P. P. Patel, L. Chang, A. J. Rivnak, E. P. Ferrell, J. D. Randall, G. K. Provuncher, D. R. Walt, and D. C. Duffy. 2010. Single-molecule enzyme-linked immunosorbent assay detects serum proteins at subfemtomolar concentrations. Nat. Biotechnol. 28:595-599. https://doi.org/10.1038/nbt.1641.

Sheng, W., T. Xu, H. Ma, X. Wang, Q. Li, and J. Li. 2009. Development of an indirect competitive enzyme-linked immunosorbent assay for detection of danofloxacin residues in beef, chicken and pork meats. Food Agric. Immunol. 20:35-47. https://doi.org/10.1080/ 09540100802657581.

Tian, E. J., C. L. Chen, W. J. Hu, Y. S. Miao, I. Muhammad, Q. M. Zhang, Y. H. Liu, L. Xu, J. X. Bao, L. J. Ding, and J. C. Li. 2019. Population pharmacokinetics for danofloxacin in the intestinal contents of healthy and infected chickens. J. Vet. Pharmacol. Ther. 42:556-563. https://doi.org/10.1111/jvp.12799.

Wen, K., G. Nolke, S. Schillberg, Z. H. Wang, S. X. Zhang, C. M. Wu, H. Y. Jiang, H. Meng, and J. Z. Shen. 2012. Improved fluoroquinolone detection in ELISA through engineering of a broad-specific single-chain variable fragment binding simultaneously to 20 fluoroquinolones. Anal. Bioanal. Chem. 403:2771-2783. https://doi.org/ 10.1007/s00216-012-6062-z.

Xiong, Y., K. Pei, Y. Wu, H. Duan, W. Lai, and Y. Xiong. 2018. Plasmonic ELISA based on enzyme-assisted etching of Au nanorods for the highly sensitive detection of aflatoxin B-1 in corn samples. Sensor. Actuat. Biol. Chem. 267:320-327. https://doi.org/10.1016/j .snb.2018.04.027.

Zhang, X., C. Wang, L. Yang, W. Zhang, J. lin, and C. Li. 2017. Determination of eight quinolones in milk using immunoaffinity microextraction in a packed syringe and liquid chromatography with fluorescence detection. J. Chromatogr. B Analyt. Technol. Biomed. Life Sci. 1064:68-74. https://doi.org/10.1016/j.jchromb 2017.09.004.

\section{ORCIDS}

Suhua Wang (ํ) https://orcid.org/0000-0002-4554-5923 